\title{
A Prospective Controlled Trial to Evaluate Safety and Efficacy of in vitro Expanded Recipient Regulatory T Cell Therapy and Tocilizumab Together With Donor Bone Marrow Infusion in HLA-Mismatched Living Donor Kidney Transplant Recipients (Trex001)
}

OPEN ACCESS

Edited by:

Carole Guillonneau,

INSERM U1064 Centre de Recherche

en Transplantation et

Immunologie, France

Reviewed by:

Antoine Durrbach

Hôpital Kremlin-Bicêtre, France

Ron Shapiro,

Mount Sinai Hospital, United States

${ }^{*}$ Correspondence:

Thomas Wekerle

thomas.wekerle@meduniwien.ac.at

Specialty section:

This article was submitted to

Translational Medicine,

a section of the journal

Frontiers in Medicine

Received: 27 November 2020 Accepted: 31 December 2020

Published: 27 January 2021

Citation:

Oberbauer R, Edinger $M$,

Berlakovich G, Kalhs P, Worel N,

Heinze G, Wolzt M, Lion T and

Wekerle T (2021) A Prospective Controlled Trial to Evaluate Safety and

Efficacy of in vitro Expanded Recipient

Regulatory T Cell Therapy and

Tocilizumab Together With Donor

Bone Marrow Infusion in

HLA-Mismatched Living Donor Kidney

Transplant Recipients (Trex001).

Front. Med. 7:634260.

doi: 10.3389/fmed.2020.634260
Rainer Oberbauer ${ }^{1}$, Matthias Edinger ${ }^{2}$, Gabriela Berlakovich ${ }^{3}$, Peter Kalhs ${ }^{4}$, Nina Worel ${ }^{5}$, Georg Heinze ${ }^{6}$, Michael Wolzt ${ }^{7}$, Thomas Lion ${ }^{8,9,10,11}$ and Thomas Wekerle ${ }^{12 *}$

${ }^{1}$ Division of Nephrology and Dialysis, Department of Internal Medicine III, Medical University of Vienna, Vienna, Austria, ${ }^{2}$ University Hospital Regensburg, Department of Internal Medicine III \& Regensburg Center for Interventional Immunology (RCl), Regensburg, Germany, ${ }^{3}$ Division of Transplantation, Department of Surgery, Medical University of Vienna, Vienna, Austria, ${ }^{4}$ Bone Marrow Transplant Unit, Department of Internal Medicine I, Medical University of Vienna, Vienna, Austria, ${ }^{5}$ Department of Blood Group Serology and Transfusion Medicine, Medical University of Vienna, Vienna, Austria, ${ }^{6}$ Section for Clinical Biometrics, Center for Medical Statistics, Informatics and Intelligent Systems, Medical University of Vienna, Vienna, Austria, ${ }^{7}$ Clinical Trials Coordination Centre, Medical University of Vienna, Vienna, Austria, ${ }^{8}$ St. Anna Children's Cancer Research Institute, Vienna, Austria, ${ }^{9}$ Ludwig Boltzmann Institute for Hematology \& Oncology, Medical University of Vienna, Vienna, Austria, ${ }^{10}$ Labdia Labordiagnostik GmbH, Vienna, Austria, ${ }^{11}$ Department of Pediatrics, Medical University of Vienna, Vienna, Austria, ${ }^{12}$ Section of Transplantation Immunology, Division of Transplantation, Department of Surgery, Medical University of Vienna, Vienna, Austria

Background: The induction of donor-specific immunological tolerance could improve outcome after kidney transplantation. However, no tolerance protocol is available for routine clinical use. Chimerism-based regimens hold promise, but their widespread application is impeded in part by unresolved safety issues. This study tests the hypothesis that therapy with polyclonal recipient regulatory T cells (Tregs) and anti-IL6R (tocilizumab) leads to transient chimerism and achieves pro-tolerogenic immunomodulation in kidney transplant recipients also receiving donor bone marrow $(\mathrm{BM})$ without myelosuppressive conditioning of the recipient.

Methods/design: A prospective, open-label, controlled, single-center, phase I/lla academic study is performed in HLA-mismatched living donor kidney transplant recipients.

Study group: Recipients of the study group receive in vitro expanded recipient Tregs and a donor bone marrow cell infusion within 3 days after transplantation and tocilizumab for the first 3 weeks post-transplant. In addition they are treated with thymoglobulin, belatacept, sirolimus, and steroids as immunosuppression. Starting 6 months post-transplant, sirolimus and steroids are withdrawn in a step-wise manner in stable patients. 
Control group: Recipients of the control group are treated with thymoglobulin, belatacept, sirolimus, and steroids as immunosuppression. Co-primary endpoints of safety (impaired graft function [eGFR $<35 \mathrm{~mL} / \mathrm{min} / 1.73 \mathrm{~m}^{2}$ ], graft-vs.-host disease or patient death by 12 months) and efficacy (total leukocyte donor chimerism within 28 days post-transplant) are assessed. Secondary endpoints include frequency of biopsy-proven acute rejection episodes and subclinical rejection episodes on surveillance biopsies, assessment of kidney graft function, and the evaluation whether the study protocol leads to detectable changes in the immune system indicative of pro-tolerogenic immune modulation.

Discussion: The results of this trial will provide evidence whether treatment with recipient Tregs and donor $\mathrm{BM}$ is feasible, safe and efficacious in leading to transient chimerism. If successful, this combination cell therapy has the potential to become a novel treatment option for immunomodulation in organ transplantation without the toxicities associated with myelosuppressive recipient conditioning.

Trial registration: European Clinical Trials Database EudraCT Nr 2018-003142-16 and clinicaltrials.gov NCT03867617.

Keywords: kidney transplantation, cell therapy, regulatory T cells, chimerism, tolerance, bone marrow, belatacept, tocilizumab

\section{INTRODUCTION}

Long-term outcome after kidney transplantation has improved little over the last decades and immune-mediated injury remains a leading cause of graft loss despite modern immunosuppressive drug therapy (1). The establishment of donor-specific immunological tolerance would be a solution to this problem $(2,3)$. Chimerism-based tolerance, established through the co-transplantation of hematopoietic stem cells together with a kidney from the same donor, has emerged from extensive preclinical research as a promising approach for clinical translation $(3,4)$. Chimerism was originally observed to lead to tolerance toward the hematopoietic cell donor in naturally occurring freemartin cattle that exchange hematopoietic cells intrauterinely through a shared placental circulation (5). Subsequently, Medawar and colleagues actively induced transplant tolerance by transferring donor hematopoietic cells (6). Over the ensuing decades, the mechanisms contributing to donor-specific tolerance have been delineated in increasing detail. Intrathymic clonal deletion of emerging donor-reactive thymocytes is a key feature of chimerism-based tolerance $(7,8)$ and one reason why tolerance induced through chimerism typically is very robust. Extrathymic clonal deletion of mature donor-reactive $\mathrm{T}$ cells also plays a role, at least in some chimerism protocols (9). Notably, regulatory mechanisms have more recently been recognized as essential mechanisms in chimerism-based tolerance $(10,11)$. The interplay of deletional and regulatory mechanisms seems to lead to the most durable states of tolerance in pre-clinical chimerism models (4). A cardinal feature of chimerism-based tolerance is its successful translation to large animal models, including non-human primates (3). Besides, anectodal cases of patients that had undergone hematopoietic stem cell transplantation for a conventional indication and that subsequently accepted a kidney graft from the original stem cell donor without immunosuppression provided proof-of-concept that chimerism can also lead to tolerance in humans (2).

Three pilot trials have recently been conducted that investigated the simultaneous co-transplantation of donor hematopoietic stem cells (donor BM or mobilized peripheral blood stem cells [mPBSC]) for the purpose of tolerance induction in living donor kidney transplant recipients (12-16). Tolerance was achieved in $40-60 \%$ of HLA-mismatched transplants in two trials and the rate of tolerance was even higher in the HLA-identical kidney transplant setting (13). The protocols of the three trials differ in important aspects, but all have in common that they involve myelosuppressive conditioning (i.e., irradiation of the recipient and/or cytotoxic drug treatment). Total lymphoid irradiation $(10 \times 80-120 \mathrm{cGy})$ was part of the Stanford protocol (13), $700 \mathrm{cGy}$ thymic irradiation together with cyclophosphamide was given in the MGH protocol (12) and 200 cGy total body irradiation together with cyclophosphamide and fludarabine in the Northwestern protocol (14). Myelosuppression led to transient leukopenia [absolute neutrophil count $<500 / \mu \mathrm{L}$ for $\approx 12$ days in the two HLA-mismatched cohorts (MGH and Northwestern) $(12,14)]$ and was associated with infectious complications in some cases (17). An engraftment syndrome that was linked to recovery of recipient hematopoiesis following myelosuppression, occurred in all patients in the MGH trial (12). Myelosuppression is considered problematic by many clinicians for use outside of clinical trials in specialized centers. Graftvs.-host disease (GVHD) occurred in the Northwestern trial (but not the other two trials), leading to one fatality (17). GVHD is widely viewed to be an unacceptable complication in 
living-donation kidney transplantation and needs to be strictly avoided. Collectively, these landmark trials provide proof-ofconcept that chimerism-based tolerance can be achieved in the clinical setting, but routine application of the current protocols in kidney transplant recipients is impeded by safety issues.

A key challenge in the field therefore is to develop a chimerism-based tolerance protocol that is sufficiently safe for more extensive clinical application, but still efficacious. Pre-clinical and clinical data indicate that very low levels of chimerism can suffice for tolerance induction. Besides, chimerism does not need to be permanent but can be transient $(12,18)$, which can be achieved with milder conditioning regimens. In a series of rodent studies, costimulation blockade (9), mTOR inhibition (19), and IL6 blockade (20) were identified as treatments that allowed conditioning requirements to be reduced. Myelosuppression, however, remained an indispensable factor for achieving engraftment of clinically obtainable BM doses with such protocols. In contrast, the administration of polyclonal recipient Tregs promoted engraftment of fully mismatched BM in the absence of myelosuppression (10). Hematopoietic stem cell engraftment (tested by secondary BM transplantation [BMT]) was achieved across full MHC-barriers in recipients otherwise conditioned only with a short-course of costimulation blockade and the mTOR inhibitor rapamycin. This effect was observed with several types of Tregs (i.e., in vitro activated $\mathrm{CD} 4{ }^{+} \mathrm{CD} 25^{+}$Tregs, TGF $\beta$-induced Tregs and FoxP3-transduced Tregs) (10). Levels of multi-lineage chimerism were low, but the state of tolerance observed was more complete compared to previous myelosuppressive rodent regimens (achieving higher chimerism levels) with regard to several endpoints (21-23). Recently, the BM engraftmentpromoting effect of polyclonal recipient Tregs has been confirmed in a small non-human primate (NHP) study (24), in which Treg application extended overall chimerism and led to chimerism within the T cell lineage. Treg cell therapy is currently explored as promising therapy in several immunological disorders, including the prevention of transplant rejection (2527) and the prevention of GVHD (28-30). Preliminary results indicate that Treg therapy induced tolerance in a cohort of livingdonor liver transplant recipients (31), but the same therapy failed in kidney transplantation (32). The full potential of Treg therapy alone for inducing tolerance in organ transplantation thus still needs to be determined.

The present study investigates combination cell therapy with recipient Tregs and donor BM, together with IL6 pathway blockade, as a potential strategy for inducing transient chimerism and pro-tolerogenic immunomodulation in kidney transplantation (the study concept is summarized in Figure 1).

\section{METHODS AND ANALYSIS}

\section{Protocol Version}

Version 2.0; December 4, 2018.

\section{Trial Sponsor and Role of Sponsor}

The Department of Surgery, Medical University of Vienna (Spitalgasse 23, 1090 Vienna, Austria) is the trial sponsor. The

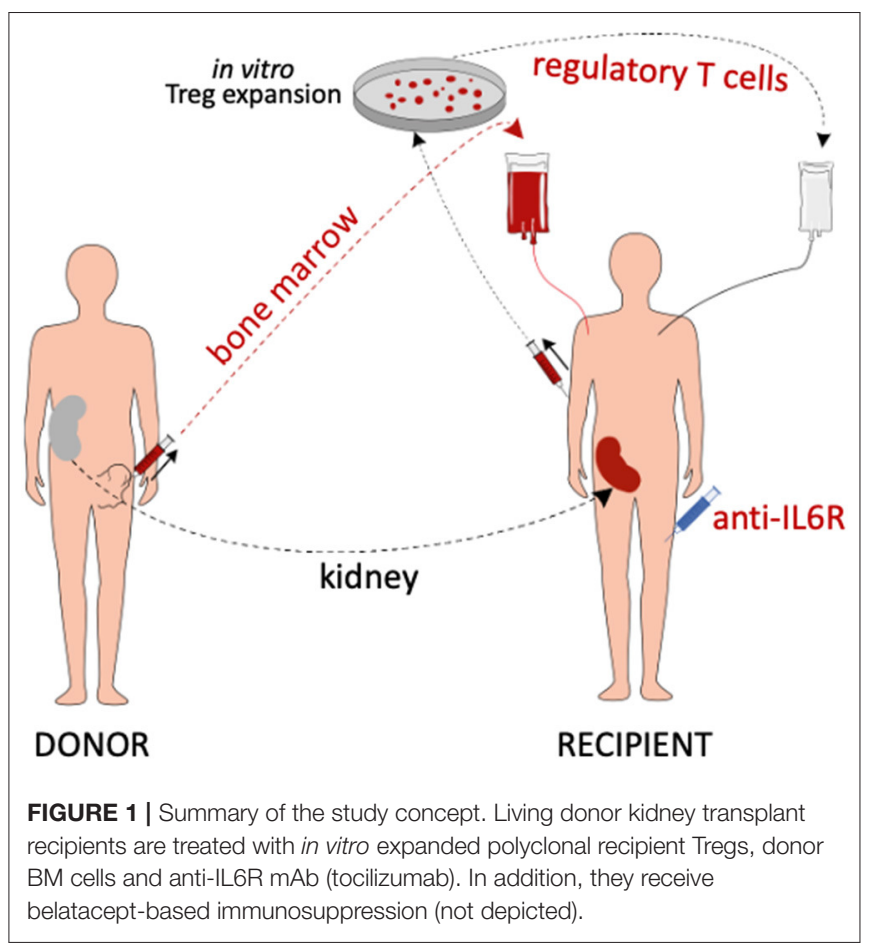

study sponsor has no role in the design of the study or the analysis and publication of its results.

\section{Ethics Approval}

The study has been approved by the ethics committee of the Medical University of Vienna (Ethikkommission Medizinische Universität Wien, EK Nr: 1871/2018) and the Austrian Federal Office for Safety in Health Care (BASG Bundesamt für Sicherheit im Gesundheitswesen, Verfahrensnummer 11337515).

\section{Trial Design}

Prospective, open-label, controlled, single-center, phase I/IIa study.

\section{Subject Population}

Subjects are recruited from patients scheduled to receive a living donor kidney transplant at the Vienna General Hospital/Medical University of Vienna.

\section{Eligibility Criteria}

\section{Inclusion and Exclusion Criteria}

The donor and the recipient need to provide written informed consent. The inclusion and exclusion criteria that need to be fulfilled for enrolment into the trial are the same for recipients in the study group and recipients in the control group. Inclusion/exclusion criteria for donors in the study group are distinct from those for donors in the control group. Inclusion and exclusion criteria are listed in Table $\mathbf{1}$.

\section{Interventions \\ Study Group Treatment}

The study group receives in vitro expanded polyclonal recipient Tregs (RegTivex), anti-IL6R (tocilizumab), and 
TABLE 1 | Inclusion and exclusion criteria for recipients and donors of the study and the control group.

Inclusion criteria

For recipients (study and control group):

- Patient has provided written informed consent

- Patient is 18 years or older

- Patient is a planned recipient of a living donor kidney transplant

- Patient is a planned recipient of an ABO blood group-compatible kidney graft

- Patient is a planned recipient of a kidney graft from a donor that is not HLA-identical

- Patient is negative for DSA

- WOCBP must have a negative pregnancy test at inclusion

- WOCBP must be using an adequate method of contraception to avoid pregnancy throughout the study and for up to 12 weeks after the study in such a manner that the risk of pregnancy is minimized

For donors (study group only):

- Participant has provided written informed consent

- Participant is 18 years or older

- Participant is suitable to donate bone marrow according to the guidelines of the Department of Blood Group Serology and Transfusion Medicine

- WOCBP must not be pregnant at inclusion (i.e., negative pregnancy test)

For donors (control group only):

- Participant has provided written informed consent to donate blood for immune monitoring analyses

- Participant is 18 years or older

Exclusion criteria

For recipients (study and control group):

- Patient is EBV-negative on serology

- Patient is HIV positive or suffering from chronic viral hepatitis

- Patient is CMV negative and receiving a kidney from a CMV-positive donor

- Positive T-cell lymphocytotoxic cross match

- Patient with prior kidney transplant or non-renal solid organ transplant

- Patient has a known contraindication to any of the protocol-specified treatments

- Patient had been diagnosed with a malignancy within 5 years prior to study entry, excluding non-metastatic basal or squamous cell carcinoma of the skin

- Female patients who are breast-feeding

- Female patients with a positive pregnancy test at the time of evaluation

donor $\mathrm{BM}$, in addition to belatacept-based immunosuppression (Figure 2A). Tregs and BM are administered within 3 days after kidney transplantation. Anti-IL6R antibody (tocilizumab) is administered weekly for the first 3 weeks post-transplant. In addition, patients receive immunosuppressive drug therapy consisting of induction therapy with anti-thymocyte globulin (Thymoglobulin) and maintenance therapy with belatacept, sirolimus, and steroids. Starting 6 months post-transplant, sirolimus and steroids are withdrawn in a step-wise manner in stable patients. Sirolimus weaning starts first, to be followed by weaning of steroids 3 months later. By 12 months, stable patients are receiving belatacept monotherapy. In vitro expanded regulatory T cells (RegTivex) and tocilizumab (RoActemra; antiIL6R monoclonal antibody) are the investigational medicinal products (IMPs) of this study.

\section{Control Group Treatment}

The control group receives the same immunosuppressive drug combination as the study group consisting of thymoglobulin, belatacept, sirolimus, and steroids (Figure 2B). No Tregs and no anti-IL6R are given and no BM is administered. No withdrawal of sirolimus or steroids is attempted.

\section{Immune Monitoring}

Extensive immune monitoring accompanies the study with the aim to yield information about the activity and the mode of action of the treatment protocol (Figure 2C). Samples
(PBMC, serum and DNA) are collected from donors and recipients of both groups at pre-defined time points and are also biobanked for future analysis. A range of laboratory assays have been developed for use in transplant patients. These assays can provide important scientific information on the efficacy of immunomodulatory protocols, but they have to be considered experimental and in large parts are not validated or approved for clinical diagnostics. Results from these assays are not used for clinical decisions. Immune monitoring is planned to include the assessment of lineage-specific chimerism in FACS-sorted cell subsets using the Droplet Digital (dd) PCR technology, leukocyte phenotyping by multi-color flow cytometry, functional $\mathrm{T}$ cell assays (IFN $\gamma$-ELISPOT, $\mathrm{T}$ cell proliferation, and $\mathrm{T}$ cell suppressive function), next generation sequencing (NGS)-based $\mathrm{T}$ cell receptor (TCR) repertoire analysis of the Treg product and of recipient $\mathrm{T}$ cells in blood and in the graft, and intragraft gene expression analysis.

\section{Randomization}

No randomization is performed. The complex design which requires recipient leukapheresis plus thymoglobulin treatment 2 weeks prior to transplant and cell therapy with Tregs and BM in the recipient and BM harvesting from the donor in the study group but not the control group, would impede enrolment within a reasonable time frame, if randomization were performed, as it is assumed that more subjects are willing to participate in 


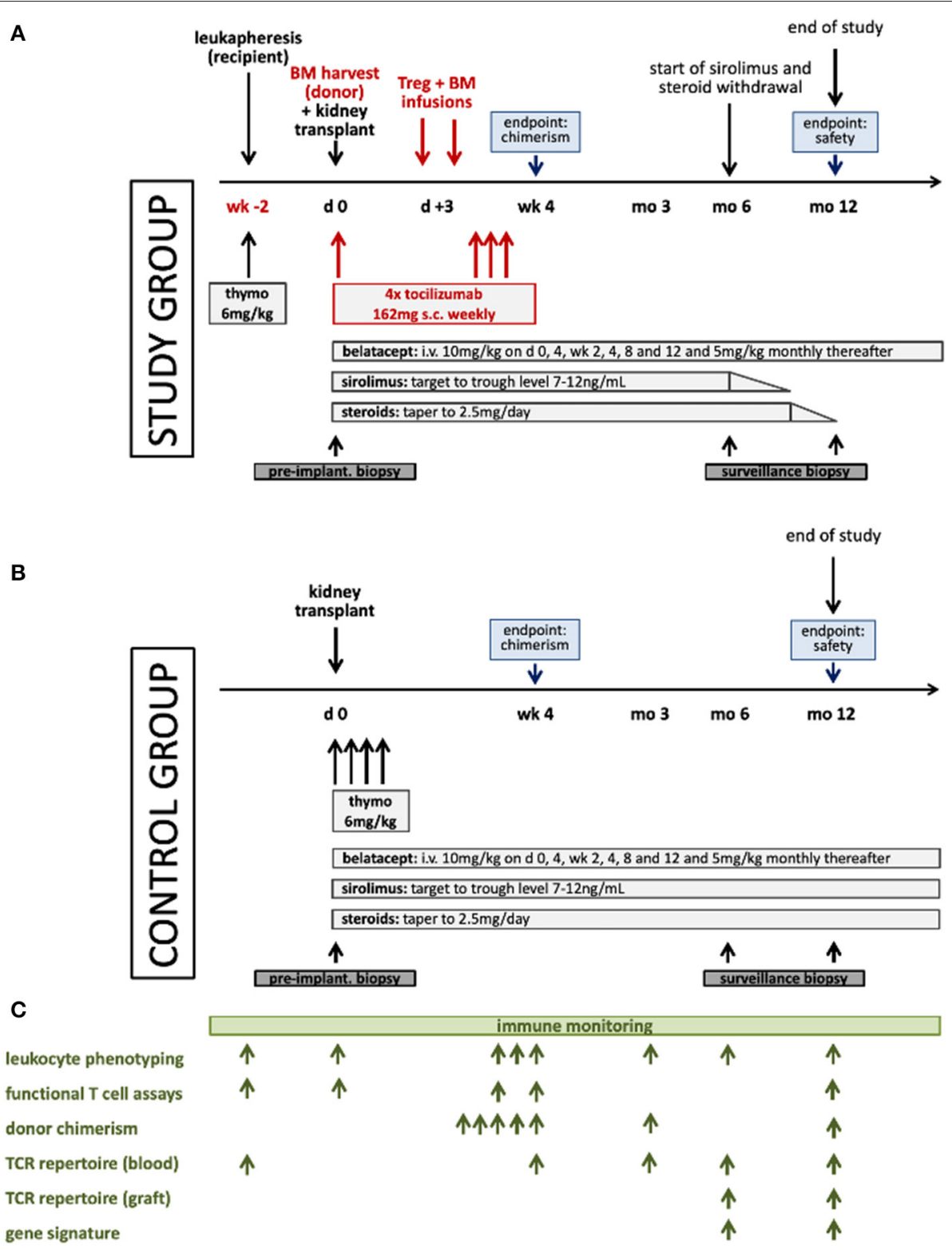

FIGURE 2 | Schematic illustration of the treatment protocols for the study (A) and the control (B) groups and the immune monitoring plan (C). Thymo denotes thymoglobulin.

the control than the study group. Due to the small sample size, randomization would require stratification, as otherwise outcome predictors would likely be unevenly distributed between groups. Due to the number of living donor transplants performed annually at the Medical University of Vienna, stratification would likely make enrollment in this single center study infeasible. Therefore, also no attempt at matching the number of HLAdisparities between the groups is undertaken. Thus, mainly for reasons of feasibility no randomization is performed.

\section{Treg Cell Product}

The IMP consists of in vitro expanded CD $45 \mathrm{RA}^{+} \mathrm{CD} 4^{+} \mathrm{CD} 25^{\text {high }} \mathrm{CD} 127^{\text {low } / \text { neg }}$ regulatory $\mathrm{T}$ cells isolated from a leukapheresis product of the kidney transplant recipient. The Treg product (RegTivex) is generated at the José-Carreras-Centrum for Somatic Cell Therapy (JCC; www.carreras-centrum.de) at the University Hospital Regensburg (Germany). Two weeks prior to the scheduled kidney transplant, the recipient undergoes standard leukapheresis at the Medical University of Vienna. The time point of leukapheresis is chosen to allow optimum Treg expansion until the scheduled day of infusion. The leukapheresis product is shipped to the JCC using validated transport conditions, enriched for $\mathrm{CD}_{2} 5^{+}$cells using bead selection technologies, then stained for CD4, CD25, CD127, and CD45RA and FACS-sorted for the CD45RA-positive Treg subpopulation (33). Cells are then in vitro expanded for 
$\sim 2$ weeks (2-3 log expansion rates) using CD3/28 stimulation beads and high-dose rh-IL-2 (34). After bead-removal and quality-controlled product release, the Treg cell product is shipped back to Vienna. The cell product is infused within $48 \mathrm{~h}$ after release (note: Regensburg is a 4-h drive away from Vienna). Kidney transplant recipients of the study group are infused intravenously with the fresh Treg product within 3 days after the kidney transplant. The Treg and BM cell infusions are both given within 3 days post-transplant, but are administered sequentially with a time interval in-between to assess immediate infusion-related side effects individually. At this time, all kidney transplant recipients are routinely treated in-hospital at the transplant center. Treg therapy in this trial constitutes an autologous cell infusion.

The target dose for the Treg cell product is $1.0 \times 10^{7}$ cells $/ \mathrm{kg}$ body weight. This is the dose for which preliminary safety data exist from GVHD studies. The actual dose of available Tregs $/ \mathrm{kg}$ body weight will depend on several factors, including the cell number and composition of the leukapheresis product, the efficiency of the Treg expansion and the weight of the recipient. In case the target cell dose cannot be reached, doses as low as $0.3 \times 10^{7}$ cells $/ \mathrm{kg}$ body weight are permissible. This lower limit is set to minimize the risk that Treg cells from the recipient are discarded without clinical use. The maximum dose of Tregs administered is $1.5 \times 10^{7}$ cells $/ \mathrm{kg}$ body weight. The cells are administered post-operatively as a single intravenous infusion, no later than 3 days after the kidney transplant (no later than D3).

\section{Donor Bone Marrow}

Unseparated donor BM cells are used as they were shown to have advantages over mPBSC in pre-clinical experiments and as they were used successfully in a clinical pilot trial of chimerismbased tolerance $(12,15)$. The target dose is $2-3 \times 10^{8}$ nucleated cells/kg body weight (corresponding to a CD34 ${ }^{+}$cell dose of 2-3 $\times 10^{6} / \mathrm{kg}$ body weight). Similar doses have been used previously in kidney transplant recipients $(12,15)$. In case of a minor ABOincompatibility, the BM is plasma-depleted. The unseparated donor bone marrow cells are administered post-operatively as a single intravenous infusion, no later than 3 days after the kidney transplant (no later than D3). Donor bone marrow is only infused in patients that have successfully received a Treg infusion.

\section{Tocilizumab}

Tocilizumab is administered as four subcutaneous injections of $162 \mathrm{mg}$ each, starting on the day of transplant (D0) and subsequent doses on D5, D15, and D21 post-transplant. This dosing regimen corresponds to the dosing frequency approved for long-term maintenance therapy in rheumatoid arthritis.

\section{Anti-thymocyte Globulin of Rabbit Origin (Thymoglobulin)}

Thymoglobulin is administered intravenously at a dose of 6 $\mathrm{mg} / \mathrm{kg}$ body weight. In the control group, thymoglobulin is given at the time of transplant, in four divided doses, as previously reported (35). In the study group, the thymoglobulin administration needs to be moved to an earlier time point, so that thymoglobulin does not deplete the infused Tregs.
Besides, it can only be administered after leukapheresis (as the leukapheresis product would otherwise be depleted of the T cell subset necessary for Treg expansion). Therefore, thymoglobulin is administered immediately following leukapheresis, 2 weeks prior to the kidney transplant. Thereby, serum levels of active thymoglobulin (i.e., the fraction that binds to lymphocytes) are low at the time of Treg infusion (36), while numbers of lymphocytes (including $\mathrm{T}$ cells) are depleted profoundly. Two weeks after infusion of $6 \mathrm{mg} / \mathrm{kg}$ thymoglobulin the level of active thymoglobulin is expected to have fallen to or near the subtherapeutic level $(<1 \mu \mathrm{g} / \mathrm{mL})$ (36), while CD4 T cells are depleted by $>95 \%$ at this time point (36). A reduction in the number of recipient $\mathrm{T}$ cells is considered beneficial or even necessary for the efficacy (activity) of Treg therapy (1). Moreover, the depletion of recipient $\mathrm{T}$ cells promotes BM engraftment in rodent and non-human primate studies.

\section{Belatacept}

Belatacept, a CTLA4Ig derivative, is the only clinically approved alternative to calcineurin-inhibitors (CNIs) as primary immunosuppressant. CNIs impeded outcome in pre-clinical costimulation blockade-based chimerism models (19). Belatacept/CTLA4Ig, in contrast, were associated with pro-tolerogenic mechanisms and promoted BM engraftment in rodent and NHP studies (18). CTLA4Ig was also part of the pre-clinical myelosuppression-free Treg-induced chimerism protocol on which this clinical trial is based (10). The mechanistic interaction of belatacept with Tregs is complex and incompletely understood. Several papers suggest that belatacept might negatively interfere with endogenous Tregs. However, other lines of evidence, including clinical data show a neutral or even favorable effect of belatacept on Tregs $(37,38)$. The clinically approved dosing regimen of belatacept is used. Belatacept is given as intravenous infusions at a dose of $10 \mathrm{mg} / \mathrm{kg}$ on D0, D4, D13, D27, week 8 and 12 and at a dose of $5 \mathrm{mg} / \mathrm{kg}$ every 4 weeks thereafter.

\section{Sirolimus}

mTOR inhibition through sirolimus promotes Tregs while inhibiting effector $\mathrm{T}$ cells. In addition, sirolimus promoted the engraftment of allogeneic BM in preclinical studies $(10,19,39)$. Sirolimus is dosed to maintain trough levels of $7-12 \mathrm{ng} / \mathrm{mL}$, as in Ferguson et al. (35).

\section{Steroids}

Corticosteroids (prednisone, prednisolone, methylprednisolone, dexamethasone) are dosed according to local practice at the Medical University of Vienna for 12 months in the control group and 9 months in the study group.

\section{BM Harvest}

Donor BM (not more than $20 \mathrm{~mL} / \mathrm{kg}$ body weight; maximum volume $1,500 \mathrm{~mL}$ ) is obtained from the iliac crest under general anesthesia during the donor nephrectomy surgery (D0). Intraoperative BM harvesting during living donor 
nephrectomy was reported to be feasible and safe by several groups (40-43).

\section{Sirolimus and Steroid Withdrawal in Study Group}

Starting 6 months post-transplant, sirolimus and steroids are withdrawn in a step-wise manner in stable patients of the study group. Pre-defined criteria for starting sirolimus and steroid withdrawal are: absence of immunological injury on 6-month surveillance biopsy (i.e., freedom from $\mathrm{T}$ cell-mediated rejection and antibody-mediated rejection; borderline score acceptable) and absence of donor-specific antibodies (DSA). Sirolimus weaning starts first, to be followed by weaning of steroids 3 months later.

\section{Study Drug Interruption or Discontinuation}

The Investigator must temporarily interrupt or permanently discontinue the study drug if continued administration of the study drug is believed to be contrary to the best interests of the patient. The interruption or premature discontinuation of study drug might be triggered by an Adverse Event (AE), a diagnostic or therapeutic procedure, an abnormal assessment (e.g., laboratory abnormalities), or for administrative reasons, in particular withdrawal of the patient's consent. The reason for study drug interruption or premature permanent discontinuation must be documented in the CRF.

\section{Outcome}

Primary Objective:

- 1) To examine the safety of Treg therapy together with tocilizumab and donor bone marrow in living donor kidney transplant recipients.

- 2) To assess chimerism levels within the first month post-transplant.

Secondary Objectives:

- To demonstrate that the study protocol allows the initiation of a step-wise reduction of immunosuppression up to a point when patients receive drug monotherapy.

- To gain insight as to whether the study protocol leads to detectable changes in the immune system indicative of protolerogenic immune modulation.

- To assess the frequency of biopsy-proven acute rejection episodes.

- To assess the frequency of subclinical rejection episodes on surveillance biopsies.

- To assess kidney graft function.

- To assess the area-under-the-curve (AUC) of chimerism.

The composite safety endpoint is defined as the incidence of GVHD, impaired graft function [eGFR $<35 \mathrm{~mL} / \mathrm{min} / 1.73 \mathrm{~m}^{2}$ ] or patient death, whichever occurs first, within the first 12 months post-transplantation (co-primary endpoint 1 ).

The efficacy endpoint is defined as individual peak chimerism levels observed in each patient within 4 weeks post-transplant (co-primary endpoint 2). Total leukocyte donor chimerism will be measured by sensitive ddPCR technology at days 5, 10, 15, 21, and 28 post-transplant in both groups (44).

\section{Sample Size}

Six patients (recipients) per group eligible for per-protocol analysis, up to 10 patients per group maximum. For each recipient one donor is enrolled in the same group.

The sample size is based primarily on feasibility to allow enrollment within a reasonable time frame. Beyond this premise, sample size calculation yielded a number of six for the study group to conclude with a probability (statistical power) of 0.8 that the incidence of the safety endpoint is not $>65 \%$ (co-primary endpoint 1 ) at a one-sided significance level of $2.5 \%$ (one-sided test for binomial proportion). This margin of $65 \%$ is accepted for statistical analysis to preserve feasibility. A more robust safety analysis will only be possible in potential larger subsequent trials. By including also six patients in the control group the study has $86.5 \%$ power to detect a difference in mean peak chimerism on the logarithmic-base- 2 scale of 2 log-units (a 4 -fold difference in geometric means) given an assumed standard deviation of 1 log-unit (co-primary endpoint 2). No data are available from which a meaningful assumption on the standard deviation of mean peak chimerism (on the logarithmic-base-2 scale) could be derived. Our assumption corresponds to a ratio between upper and lower limits of a normal range (97.5th and 2.5th percentiles) of about 16 (four log units), which was considered conservative enough when discussing it among the principal investigators and the study statistician.

\section{Statistical Methods Analysis Sets}

Two different analysis sets are defined:

\section{Modified Intention-to-Treat Set}

This analysis set includes subjects who were enrolled and received at least one dose of study drug.

\section{Per-protocol Set}

This analysis set comprises all subjects who received the kidney transplant, the Treg infusion (at a minimum dose of $0.7 \times 10^{7}$ cells/kg body weight), the BM infusion and at least one dose of tocilizumab (study group), all subjects of the control group who were treated with at least one dose of belatacept, and who completed 4 weeks of follow-up and who did not violate the protocol in a way that might affect the evaluation of the effect of the study drug(s) on the primary objective, i.e., without major protocol violations.

\section{Endpoints Analysis \\ Primary Endpoint Analysis}

Co-primary Endpoint 1 (Safety). Co-primary endpoint 1 will be evaluated in the study group, using the modified intentionto-treat set. The endpoint will be summarized by computing the proportion of patients reaching co-primary endpoint 1 and the corresponding two-sided 95\% exact (Clopper-Pearson) confidence interval.

Null hypothesis: The incidence of the composite safety endpoint (GVHD, impaired graft function [eGFR $<35$ $\mathrm{mL} / \mathrm{min} / 1.73 \mathrm{~m}^{2}$ ] or patient death by 12 months post-transplant) in treated patients is $65 \%$ or higher. 
Alternative hypothesis: The incidence of the composite safety endpoint (GVHD, impaired graft function [eGFR $<35$ $\mathrm{mL} / \mathrm{min} / 1.73 \mathrm{~m}^{2}$ ] or patient death by 12 months post-transplant) in treated patients is $<65 \%$.

For assessing impaired graft function (eGFR $<35$ $\mathrm{mL} / \mathrm{min} / 1.73 \mathrm{~m}^{2}$ ) eGFR values after the first month posttransplant will be considered (to exclude low eGFR values due to delayed graft function in the immediate post-transplant period). eGFR is calculated according to the CKD-EPI (Chronic Kidney Disease Epidemiology Collaboration) formula. GVHD diagnosis needs to be confirmed by histology.

The proportion will be statistically tested against the null hypothesis of the proportion being $65 \%$ or greater using a one-sample test for the binomial proportion at a one-sided significance level of 0.025 . The null hypothesis will not be rejected (corresponding to a negative study result) if more than one out of the six patients experiences the safety endpoint, as then the upper limit of the one-sided $97.5 \%$ confidence interval for the safety endpoint incidence will reach beyond $65 \%$.

Co-primary Endpoint 2 (Efficacy). Total leukocyte donor chimerism is measured by sensitive ddPCR technology on D5, D10, D15, D21, and D 28 post-transplant in both study groups. Co-primary endpoint 2 is defined as the individual peak chimerism level, i.e., the maximum value over the five measurements taken in each patient $\left(\mathrm{C}_{\max }\right)$. The endpoint will be evaluated in the per-protocol set.

The endpoint will be summarized by computing mean and standard deviation of log-base-2 peak chimerism in both groups. Mean values of the two treatment arms will be compared using a one-sided two-sample $t$-test assuming unequal variance (Satterthwaite-Welch-test) with a significance level of 0.025 .

Null hypothesis: The expected level of chimerism $\left(\log _{2} C_{\max }\right)$ in patients treated with the experimental treatment is not higher than that of control patients.

Alternative hypothesis: The expected level of chimerism $\left(\log _{2}\right.$ $\mathrm{C}_{\max }$ ) in patients treated with the experimental treatment is higher than that of control patients.

The two null hypotheses cover different aspects and thus the two co-primary endpoints will be tested independently and both will be reported. Under the global null hypothesis and assuming independence of safety and efficacy, the one-sided probability to reject one or two null hypotheses is $5 \%$.

\section{Secondary Endpoint Analysis}

All secondary endpoints will be analyzed in a purely descriptive manner. Thus, any inferential measures, such as $p$-values and confidence intervals are only meant to describe the precision of summary statistics, but not to confirm or reject a study hypothesis. Categorical data will be expressed as frequency counts and percentages. Continuous variables will be summarized by their mean, standard deviation, minimum and maximum. For repeatedly measured outcomes, pre- and all post-baseline values will be assessed and graphically presented.

\section{Oversight and Monitoring}

Monitoring is performed by the Clinical Trials Coordination Centre of the Medical University of Vienna. A Data and Safety Monitoring Board (DSMB) that it is independent from the sponsor and has no competing interests regularly reviews the clinical data of the trial and has the authority to recommend alterations and/or termination of the trial for safety reasons.

\section{Adverse Event Reporting and Harms}

An Adverse Event (AE) is defined as any untoward adverse change from the subject's baseline condition, i.e., any unfavorable and unintended sign including an abnormal laboratory finding, symptom or disease which is considered to be clinically relevant by the physician that occurs during the course of the study, whether or not considered related to the study drug. A special section is designated to adverse events in the case report form documenting the details of the AE. The severity of clinical AEs is graded on a three-point scale: mild, moderate, severe, and reported on specific AE pages of the CRF.

For all AEs, the Investigator assesses the causal relationship between the study drug and the $\mathrm{AE}$ using his/her clinical expertise and judgment (not related-unlikely-possibly related-probably related-related).

A Serious Adverse Event (SAE) is defined by the International Conference on Harmonization (ICH) guidelines and GCP guidelines as any AE fulfilling at least one of the following criteria:

- Results in deaths.

- Life-threatening-defined as an event in which the subject was, in the judgment of the Investigator, at risk of death at the time of the event.

- Requiring subject's hospitalization or prolongation of existing hospitalization.

- Resulting in persistent or significant disability or incapacity (i.e., a substantial disruption of a person's ability to conduct normal life functions).

- Congenital anomaly or birth defect.

- Is medically significant or requires intervention to prevent at least one of the outcomes listed above.

In case of a serious adverse event, the Investigator has to use all supportive measures for best patient (recipient and donor) treatment. A written report is also to be prepared detailing the SAE. If applicable, the initial report should be followed by a follow up report, indicating the outcome of the SAE.

Suspected unexpected serious adverse reactions (SUSARs) are defined as all serious adverse reactions with suspected causal relationship to the study drug that is unexpected (not previously described in the Summary of Product Characteristics or Investigator's brochure) and serious. The regulatory authorities, and the Institutional Review Board/Independent Ethics Committee (IRB/IEC) must be informed about all SUSAR. Such reports containing all relevant detail shall be made by the sponsor.

\section{Trial Status}

Recruitment started on August 1, 2019 and is expected to be completed by April 2022. 


\section{DISCUSSION}

Several considerations informed the trial design. The trial is designed with patient safety as highest priority. There is no myelosuppression, and thus no engraftment syndrome is expected (which is related to recovering recipient hematopoiesis after myelosuppression). The risk of GVHD is minimal as it is expected that no permanent chimerism is induced. Immunosuppression is reduced to belatacept monotherapy in the study group, but complete withdrawal is not attempted in this first trial of combined Treg and BM cell therapy. Thereby the potential risk of immunological damage to the graft during immunosuppression withdrawal is avoided $(15,40)$. Unseparated donor $\mathrm{BM}$ at approximately the proposed dose (i.e., $\approx 3 \times 10^{8}$ nucleated cells/kg body weight) has been used safely (without GVHD) in several clinical transplant trials of "BM augmentation" (i.e., BM infusion without myelosuppressive conditioning) (41, 42, 45, 46). Moreover, it has been demonstrated that donor BM cell infusion does not lead to sensitization (i.e., no de novo DSA) in patients receiving belatacept-based immunosuppression (with sirolimus and alemtuzumab) (42).

While a control group was not included in the three pilot trials of chimerism-based tolerance published to date (12-14), its inclusion in the study design reported herein allows more robust conclusions to be drawn from the study. Due to the complex design which requires recipient leukapheresis and BM harvesting from the donor in the study group but not the control group, no randomization will be performed. A non-experimental immunosuppressive regimen, for which clinical data from a phase II trial exist, was selected for use in the control group, in order to minimize any risk for patients in this group and to allow an overall favorable risk-benefit assessment of the study. Therefore, also no BM infusion is given in the control group. This group will serve as reference for assessing the primary and secondary endpoints and for comparison of immune monitoring results. Since BM infusion alone (i.e., without concomitant myelosuppression or Treg therapy) was associated only with very low levels of chimerism $(41,45,46)$ or no detectable chimerism (42) in kidney recipients, the control group provides an important reference for comparing chimerism levels (coprimary endpoint 2).

The protocol is designed to achieve transient chimerism without myelosuppression. Pre-clinical data demonstrate that the administration of polycloncal recipient Tregs at the time of $\mathrm{BM}$ infusion can achieve BM engraftment in this setting. The mechanisms how this effect is mediated are not fully delineated yet, but control of donor-reactive NK cells appears to be critical (47). Blocking the IL6 pathway was also shown to promote BM engraftment in the absence of myelosuppression, albeit with less potency (20). This effect is associated with an increase in the frequency of endogenous Tregs. Therefore, in the present trial Treg therapy and tocilizumab are given at the time of BM infusion within the shortest time frame considered clinically safe. BM infusion should be given at or near the time of kidney transplantation as delayed administration is less successful and required additional conditioning in non-human primate studies (48).
The in vitro expansion of the recipient Tregs takes 2-3 weeks (34). At the time of protocol submission the cell product was only approved for administration as fresh (i.e., unfrozen) product. Therefore, leukapheresis is timed to take place 2 weeks before the scheduled kidney transplant, so that after the expansion the fresh Treg cell product can be administered immediately after kidney transplant. If frozen Treg products are available, the leukapheresis of the recipient could be performed at earlier time points and the cell product could be stored until the kidney transplant.

Thymoglobulin is included in the study protocol for two reasons: (1) belatacept universally requires induction therapy, with basiliximab having been used in the original phase II and III trials. Thymoglobulin has subsequently shown promise as induction agent in combination with belatacept in a phase II trial, in which low rates of rejection were observed (35); and (2) thymoglobulin-mediated $\mathrm{T}$ cell depletion is considered to increase the therapeutic efficacy of transferred Tregs (1) which then encounter reduced numbers of T cells/lymphocytes. Besides, recipient $\mathrm{T}$ cell depletion promotes BM engraftment. Notably, as thymoglobulin is a polyclonal antibody preparation it targets a wide range of surface molecules that are not only expressed on $\mathrm{T}$ cells, but also on other leukocyte subsets (49), and thus potentially depletes also other effector lymphocytes, thereby possibly further promoting BM engraftment.

The decision to wean patients to belatacept monotherapy instead of sirolimus monotherapy was informed by several considerations, but especially the experience with belatacept monotherapy in other trials (42) and the relatively high risk of sirolimus discontinuation due to side effects.

While the small sample size is a limitation of the trial, it will be sufficient to allow a conclusion as to whether such a combination cell therapy regimen is feasible in principle and whether this concept induces transient chimerism without myelosuppression. Together with the insight from the immune monitoring assays the results of the trial are expected to permit an informed decision as to whether a subsequent trial testing Treg therapy together with donor BM infusion should be conducted and how its design should look like.

The combination of Treg therapy and donor BM infusion has shown considerable promise in pre-clinical models. The present Trex001 trial translates this novel strategy to the clinical setting. It is expected the trial will yield valuable data regarding the potential of this approach, which eventually could become a new immunomodulatory therapy in kidney transplantation with the ultimate goal of improving longterm outcome.

\section{ETHICS STATEMENT}

The studies involving human participants were reviewed and approved by the ethics committee of the Medical University of Vienna (Ethikkommission Medizinische Universität Wien, EK Nr: 1871/2018) and the Austrian Federal Office for Safety in Health Care (BASG Bundesamt für Sicherheit im Gesundheitswesen, Verfahrensnummer 11337515). The 
patients/participants provided their written informed consent to participate in this study.

\section{AUTHOR CONTRIBUTIONS}

RO, ME, GB, PK, NW, GH, MW, TL, and TW designed the study protocol. TW developed the underlying study concept and wrote the draft of the manuscript. All authors approved the final version of the manuscript.

\section{REFERENCES}

1. Wekerle T, Segev D, Lechler R, Oberbauer R. Strategies for longterm preservation of kidney graft function. Lancet. (2017) 389:215262. doi: 10.1016/S0140-6736(17)31283-7

2. Eder M, Schwarz C, Kammer M, Jacobsen N, Stavroula ML, Cowan MJ, et al. Allograft and patient survival after sequental HSCT and kidney transplantation from the same donor-a multicenter analysis. Am J Transplant. (2019) 19:475-87. doi: 10.1111/ajt.14970

3. Oura T, Cosimi AB, Kawai T. Chimerism-based tolerance in organ transplantation: preclinical and clinical studies. Clin Exp Immunol. (2017) 189:190-6. doi: 10.1111/cei.12969

4. Mahr B, Granofszky N, Muckenhuber M, Wekerle T. Transplantation tolerance through hematopoietic chimerism: progress and challenges for clinical translation. Front Immunol. (2017) 8:1762. doi: 10.3389/fimmu.2017.01762

5. Billingham RE, Lampkin GH, Medawar PB, Williams HL. Tolerance to homografts, twin diagnosis, and the freemartin condition in cattle. Heredity. (1952) 6:200-12. doi: 10.1038/hdy.1952.20

6. Billingham RE, Brent L, Medawar PB. Actively acquired tolerance of foreign cells. Nature. (1953) 172:603-6. doi: 10.1038/172603a0

7. Morris H, DeWolf S, Robins H, Sprangers B, LoCascio SA, Shonts $\mathrm{BA}$, et al. Tracking donor-reactive $\mathrm{T}$ cells: evidence for clonal deletion in tolerant kidney transplant patients. Sci Transl Med. (2015) 7:272ra10. doi: 10.1126/scitranslmed.3010760

8. Manilay JO, Pearson DA, Sergio JJ, Swenson KG, Sykes M. Intrathymic deletion of alloreactive $\mathrm{T}$ cells in mixed bone marrow chimeras prepared with a nonmyeloablative conditioning regimen. Transplantation. (1998) 66:96102. doi: 10.1097/00007890-199807150-00015

9. Wekerle T, Sayegh MH, Hill J, Zhao Y, Chandraker A, Swenson KG, et al. Extrathymic T cell deletion and allogeneic stem cell engraftment induced with costimulatory blockade is followed by central T cell tolerance. J Exp Med. (1998) 187:2037-44. doi: 10.1084/jem.187.12.2037

10. Pilat N, Baranyi U, Klaus C, Jaeckel E, Mpofu N, Wrba F, et al. Treg-therapy allows mixed chimerism and transplantation tolerance without cytoreductive conditioning. Am J Transplant. (2010) 10:751-62. doi: 10.1111/j.1600-6143.2010.03018.x

11. Savage TM, Shonts BA, Obradovic A, Dewolf S, Lau S, Zuber J, et al. Early expansion of donor-specific Tregs in tolerant kidney transplant recipients. JCI Insight. (2018) 3:e124086. doi: 10.1172/jci.insight.124086

12. Kawai T, Cosimi AB, Spitzer TR, Tolkoff-Rubin N, Suthanthiran M, Saidman SL, et al. HLA-mismatched renal transplantation without maintenance immunosuppression. $N$ Engl J Med. (2008) 358:353-61. doi: 10.1056/NEJMoa071074

13. Scandling JD, Busque S, Dejbakhsh-Jones S, Benike C, Millan MT, Shizuru JA, et al. Tolerance and chimerism after renal and hematopoietic-cell transplantation. N Engl J Med. (2008) 358:362-8. doi: 10.1056/NEJMoa 074191

14. Leventhal J, Abecassis M, Miller J, Gallon L, Ravindra K, Tollerud DJ, et al. Chimerism and tolerance without GVHD or engraftment syndrome in HLAmismatched combined kidney and hematopoietic stem cell transplantation. Sci Transl Med. (2012) 4:124ra28. doi: 10.1126/scitranslmed.3003509

15. Kawai T, Sachs DH, Sprangers B, Spitzer TR, Saidman SL, Zorn E, et al. Long-term results in recipients of combined HLA-mismatched kidney and

\section{FUNDING}

The trial was funded by the Vienna Science and Technology fund (WWTF) through project LS18-031.

\section{ACKNOWLEDGMENTS}

The authors thank Megan Sykes, Edward Geissler, and Josep Grinyo for helpful discussions.

bone marrow transplantation without maintenance immunosuppression. Am J Transplant. (2014) 14:1599-611. doi: 10.1111/ajt.12731

16. Busque S, Scandling JD, Lowsky R, Shizuru J, Jensen K, Waters $\mathrm{J}$, et al. Mixed chimerism and acceptance of kidney transplants after immunosuppressive drug withdrawal. Sci Transl Med. (2020) 12. doi: 10.1126/scitranslmed.aax 8863

17. Chhabra AY, Leventhal J, Merchak AR, Ildstad S. HSCT-based approaches for tolerance induction in renal transplant. Transplantation. (2017) 101:268290. doi: 10.1097/TP.0000000000001837

18. Yamada Y, Ochiai T, Boskovic S, Nadazdin O, Oura T, Schoenfeld D, et al. Use of CTLA4Ig for induction of mixed chimerism and renal allograft tolerance in nonhuman primates. Am J Transplant. (2014) 14:270412. doi: $10.1111 /$ ajt.12936

19. Blaha P, Bigenzahn S, Koporc Z, Schmid M, Langer F, Selzer E, et al. The influence of immunosuppressive drugs on tolerance induction through bone marrow transplantation with costimulation blockade. Blood. (2003) 101:288693. doi: 10.1182/blood-2002-10-3014

20. Granofszky N, Farkas AM, Muckenhuber M, Mahr B, Unger L, Maschke S, et al. Anti-interleukin-6 promotes allogeneic bone marrow engraftment and prolonged graft survival in an irradiation-free murine transplant model. Front Immunol. (2017) 8:821. doi: 10.3389/fimmu.2017.00821

21. Pilat N, Granofszky N, Wekerle T. Combining adoptive Treg transfer with bone marrow transplantation for transplantation tolerance. Curr Transplant Rep. (2017) 4:253-61. doi: 10.1007/s40472-017-0164-7

22. Pilat N, Farkas AM, Mahr B, Schwarz C, Unger L, Hock K, et al. Tregulatory cell treatment prevents chronic rejection of heart allografts in a murine mixed chimerism model. J Heart Lung Transplant. (2014) 33:42937. doi: 10.1016/j.healun.2013.11.004

23. Pilat N, Mahr B, Unger L, Hock K, Schwarz C, Farkas A, et al. Incomplete clonal deletion as prerequisite for tissue-specific minor antigen tolerization. JCI Insight. (2016) 1:e85911. doi: 10.1172/jci.insight.85911

24. Duran-Struuck R, Sondermeijer HP, Buhler L, Alonso-Guallart P, Zitsman $\mathrm{J}$, Kato $\mathrm{Y}$, et al. Effect of ex vivo-expanded recipient regulatory $\mathrm{T}$ cells on hematopoietic chimerism and kidney allograft tolerance across MHC barriers in cynomolgus macaques. Transplantation. (2017) 101:27483. doi: 10.1097/TP.0000000000001559

25. Sawitzki B, Harden PN, Reinke P, Moreau A, Hutchinson JA, Game DS, et al. Regulatory cell therapy in kidney transplantation (The ONE Study): a harmonised design and analysis of seven non-randomised, single-arm, phase 1/2A trials. Lancet. (2020) 395:1627-39. doi: 10.1016/S0140-6736(20)30167-7

26. Roemhild A, Otto NM, Moll G, Abou-El-Enein M, Kaiser D, Bold $\mathrm{G}$, et al. Regulatory $\mathrm{T}$ cells for minimising immune suppression in kidney transplantation: phase I/IIa clinical trial. BMJ. (2020) 371:m3734. doi: 10.1136/bmj.m3734

27. Sanchez-Fueyo A, Whitehouse G, Grageda N, Cramp ME, Lim TY, Romano $\mathrm{M}$, et al. Applicability, safety, and biological activity of regulatory $\mathrm{T}$ cell therapy in liver transplantation. Am J Transplant. (2020) 20:112536. doi: 10.1111/ajt.15700

28. Hoffmann P, Ermann J, Edinger M, Fathman CG, Strober S. Donor-type CD4(+)CD25(+) regulatory $\mathrm{T}$ cells suppress lethal acute graft-versus-host disease after allogeneic bone marrow transplantation. J Exp Med. (2002) 196:389-99. doi: 10.1084/jem.20020399

29. Edinger M, Hoffmann P, Ermann J, Drago K, Fathman CG, Strober S, et al. $\mathrm{CD} 4{ }^{+} \mathrm{CD} 25^{+}$regulatory $\mathrm{T}$ cells preserve graft-versus-tumor activity while 
inhibiting graft-versus-host disease after bone marrow transplantation. Nat Med. (2003) 9:1144-50. doi: 10.1038/nm915

30. Di Ianni M, Falzetti F, Carotti A, Terenzi A, Castellino F, Bonifacio E, et al. Tregs prevent GVHD and promote immune reconstitution in HLA-haploidentical transplantation. Blood. (2011) 117:3921-8. doi: 10.1182/blood-2010-10-311894

31. Todo S, Yamashita K, Goto R, Zaitsu M, Nagatsu A, Oura T, et al. A pilot study of operational tolerance with a regulatory T-cell-based cell therapy in living donor liver transplantation. Hepatology. (2016) 64:63243. doi: 10.1002/hep.28459

32. Koyama I, Bashuda H, Uchida K, Seino KI, Habu S, Nakajima I, et al. A clinical trial with adoptive transfer of ex vivo-induced, donor-specific immuneregulatory cells in kidney transplantation-a second report. Transplantation. (2020) 104:2415-23. doi: 10.1097/TP.0000000000003149

33. Hoffmann P, Eder R, Boeld TJ, Doser K, Piseshka B, Andreesen R, et al. Only the $\mathrm{CD} 45 \mathrm{RA}^{+}$subpopulation of $\mathrm{CD} 4{ }^{+} \mathrm{CD} 25^{\text {high }} \mathrm{T}$ cells gives rise to homogeneous regulatory T-cell lines upon in vitro expansion. Blood. (2006) 108:4260-7. doi: 10.1182/blood-2006-06-027409

34. Hoffmann P, Eder R, Kunz-Schughart LA, Andreesen R, Edinger M. Largescale in vitro expansion of polyclonal human CD4(+)CD25high regulatory $\mathrm{T}$ cells. Blood. (2004) 104:895-903. doi: 10.1182/blood-2004-01-0086

35. Ferguson R, Grinyo J, Vincenti F, Kaufman DB, Woodle ES, Marder BA, et al. Immunosuppression with belatacept-based, corticosteroid-avoiding regimens in de novo kidney transplant recipients. Am J Transplant. (2011) 11:6676. doi: 10.1111/j.1600-6143.2010.03338.x

36. Buchler M, Longuet H, Lemoine R, Herr F, Gatault P, Thibault G, et al. Pharmacokinetic and pharmacodynamic studies of two different rabbit antithymocyte globulin dosing regimens: results of a randomized trial. Transpl Immunol. (2013) 28:120-6. doi: 10.1016/j.trim.2013.03.001

37. Ahmadi SM, Holzl MA, Mayer E, Wekerle T, Heitger A. CTLA4-Ig preserves thymus-derived T regulatory cells. Transplantation. (2014) 98:115864. doi: 10.1097/TP.0000000000000421

38. Bestard O, Cassis L, Cruzado JM, Torras J, Franquesa M, Gil-Vernet S, et al. Costimulatory blockade with mTor inhibition abrogates effector T-cell responses allowing regulatory T-cell survival in renal transplantation. Transpl Int. (2011) 24:451-60. doi: 10.1111/j.1432-2277.2011.01223.x

39. Pilat N, Klaus C, Schwarz C, Hock K, Oberhuber R, Schwaiger E, et al. Rapamycin and CTLA4Ig synergize to induce stable mixed chimerism without the need for CD40 blockade. Am J Transplant. (2015) 15:156879. doi: 10.1111/ajt.13154

40. Ciancio G, Sageshima J, Akpinar E, Gaynor JJ, Chen L, Zarak A, et al. A randomized pilot study of donor stem cell infusion in living-related kidney transplant recipients receiving alemtuzumab. Transplantation. (2013) 96:8006. doi: 10.1097/TP.0b013e3182a0f68c

41. Leventhal JR, Mathew JM, Salomon DR, Kurian SM, Suthanthiran M, Tambur A, et al. Genomic biomarkers correlate with HLA- identical renal transplant tolerance. J Am Soc Nephrol. (2013) 24:1376-85. doi: 10.1681/ASN.2013010068

42. Kirk AD, Guasch A, Xu H, Cheeseman J, Mead SI, Ghali A, et al. Renal transplantation using belatacept without maintenance steroids or calcineurin inhibitors. Am J Transplant. (2014) 14:1142-51. doi: 10.1111/ajt.12712

43. Spitzer TR, Sykes M, Tolkoff-Rubin N, Kawai T, McAfee SL, Dey BR, et al. Long-term follow-up of recipients of combined human leukocyte antigen-matched bone marrow and kidney transplantation for multiple myeloma with end-stage renal disease. Transplantation. (2011) 91:6726. doi: 10.1097/TP.0b013e31820a3068

44. Fortschegger M, Preuner S, Printz D, Poetsch AR, Geyeregger R, Pichler $\mathrm{H}$, et al. Detection and monitoring of lineage-specific chimerism by digital droplet PCR-based testing of deletion/insertion polymorphisms. Biol Blood Marrow Transplant. (2020) 26:1218-24. doi: 10.1016/j.bbmt.2020.02.016

45. Fontes P, Rao AS, Demetris AJ, Zeevi A, Trucco M, Carroll P, et al. Bone marrow augmentation of donor-cell chimerism in kidney, liver, heart, and pancreas islet transplantation. Lancet. (1994) 344:151-5. doi: 10.1016/S0140-6736(94)92756-1

46. Ciancio G, Burke GW, Garcia-Morales R, Suzart K, Rosen A, Ricordi C, et al. Effect of living-related donor bone marrow infusion on chimerism and in vitro immunoregulatory activity in kidney transplant recipients. Transplantation. (2002) 74:488-96. doi: 10.1097/00007890-200208270-00010

47. Mahr B, Pilat N, Maschke S, Granofszky N, Schwarz C, Unger L, et al. Regulatory $\mathrm{T}$ cells promote natural killer cell education in mixed chimeras. Am J Transplant. (2017) 17:3049-59. doi: 10.1111/ajt.14342

48. Hotta K, Oura T, Dehnadi A, Boskovic S, Matsunami M, Rosales $\mathrm{I}$, et al. Long-term nonhuman primate renal allograft survival without ongoing immunosuppression in recipients of delayed donor bone marrow transplantation. Transplantation. (2018) 102:e128-36. doi: 10.1097/01.tp.0000542956.25922.28

49. Popow I, Leitner J, Grabmeier-Pfistershammer K, Majdic O, Zlabinger GJ, Kundi $\mathrm{M}$, et al. A comprehensive and quantitative analysis of the major specificities in rabbit antithymocyte globulin preparations. Am J Transplant. (2013) 13:3103-13. doi: 10.1111/ajt.12514

Conflict of Interest: The authors declare that the research was conducted in the absence of any commercial or financial relationships that could be construed as a potential conflict of interest.

Copyright (c) 2021 Oberbauer, Edinger, Berlakovich, Kalhs, Worel, Heinze, Wolzt, Lion and Wekerle. This is an open-access article distributed under the terms of the Creative Commons Attribution License (CC BY). The use, distribution or reproduction in other forums is permitted, provided the original author $(s)$ and the copyright owner(s) are credited and that the original publication in this journal is cited, in accordance with accepted academic practice. No use, distribution or reproduction is permitted which does not comply with these terms. 